\title{
A Green Process for Starch Oleate Synthesis by Cryptococcus sp. MTCC 5455 Lipase and Its Potential as an Emulsifying Agent
}

\author{
Aarthy Mayilvahanan, Aparna Ramchary, Aypadurai Niraikulam, \\ Gowthaman Marichetti Kuppuswami, and Kamini Numbi Ramudu**
}

Starch oleate is synthesized in an aqueous medium using lipase from the yeast Cryptococcus sp. MTCC 5455. The optimum conditions of esterification are found at $24 \mathrm{~h}$ and $30^{\circ} \mathrm{C}$ with an oleic acid/starch molar ratio of 1:2 using $500 \mathrm{U}$ of lipase and the degree of substitution was 0.26 . Spectral techniques confirm the presence of oleate group in the modified potato starch. Scanning electron microscopic and X-ray diffraction studies also reveal the morphological and crystallographic properties of starch which are disrupted during the esterification process. Thermogravimetric analysis indicates the decrease in thermal stability of starch oleate due to the transformed structure of starch from semi crystalline to an amorphous form. The synthesized starch oleate could impart $85 \%$ stability to emulsions and has potential as an emulsifier in food sector owing to its eco-friendly preparation.

\section{Introduction}

Starch, a naturally abundant, inexpensive, renewable, and biodegradable polysaccharide plays an important role in the food industry and is also used as raw material to prepare different products for various applications. ${ }^{[1]}$ However, native starches have limited industrial use owing to their poor mechanical properties, hydrophilic nature, higher tendency toward retrogradation, low shear, and thermal resistance. ${ }^{[2]}$ Modified starches were developed to eliminate these restrictions and to improve its properties for industrial applications. Starch modification is carried out through physical methods such as high temperature or pressure and chemical methods including etherification, esterification, crosslinking, or oxidation. ${ }^{[3]}$ Among these methods, esterification by replacement of hydroxyl moieties with ester groups makes starch more hydrophobic and alters its physicochemical characteristics. ${ }^{[4]}$

A. Mayilvahanan, A. Ramchary, Dr. A. Niraikulam, Dr. G. Marichetti Kuppuswami, Dr. K. Numbi Ramudu

Department of Biochemistry and Biotechnology

CSIR-Central Leather Research Institute

Chennai 600020, India

E-mail:nrkamini@rediffmail.com; nrkamini@clri.res.in

A. Mayilvahanan

CSIR-National Environmental Engineering Research Institute (NEERI)

Chennai Zonal Laboratory

Chennai 600113 , India

DOI: $10.1002 /$ star.201700325
The esterified starch has wide use in food, plastic, pharmaceutical, and biomedical industries. $^{[5]}$ They have been used as thickener, stabilizer, emulsifier, binder of frozen foods, dry mixes, flavored toppings, and sauces in the food industry. ${ }^{[6]}$ Starchbased biomaterials are used as scaffolds for the tissue engineering of bone and cartilage, carriers for the controlled release of drugs and other bioactive agents. ${ }^{[7]}$ The esters also actas substitutes for petroleum-based plastic materials and are used to make biodegradable plastics, adhesives, cigarette filters, and metal ion absorbants. ${ }^{[8]}$

Starch esters could be prepared by catalytic reactions using acids, alkalis, or enzymes. The acid or alkali catalyzed process has several limitations in catalyst recovery, equipment corrosion and waste treatment, which affects the cost of the process. Lipase catalyzed enzymatic esterification of starch is an eco-friendly process without producing chemical wastes. ${ }^{[4]}$ Moreover, the high selectivity (chiral, positional, and functional) of lipases is advantageous because it minimizes side reactions and allows easier separation of the products. ${ }^{\left[{ }^{[]}\right.}$There are reports available on synthesis of starch esters by commercial lipases from Burkholderia cepacia, ${ }^{[7]}$ Pseudomonas sp., ${ }^{[10]}$ Candida rugosa, ${ }^{[11]}$ Thermomyces lanuginosus, ${ }^{[12]}$ C. antartica, ${ }^{[3]}$ and porcine pancreas. ${ }^{[13]}$ However, these esters were synthesized in organic solvents such as pyridine, toluene, dimethyl sulfoxide (DMSO), or in ionic liquids. The use of volatile, toxic and expensive organic solvents limits further commercialization of the process in the food and pharmaceutical sector. On the other hand, though ionic liquids have been considered as desirable solvents for the homogeneous synthesis of starch esters, their cost and solubility of substrate in ionic liquids is a limitation. ${ }^{[1]}$ Thus, it is of utmost importance to design a green and efficient process in aqueous medium for the synthesis of starch esters.

Earlier, most of the synthetic reactions were carried out in nonaqueous conditions and only few lipases known to catalyze ester synthesis in aqueous media. ${ }^{[14]}$ Among them, the lipase from Cryptococcus sp. MTCC 5455 is unique and has potential in synthesis of alkyl esters, ${ }^{[15]}$ removal of triglyceride soil from fabrics, ${ }^{[16]}$ hydrolysis of oils, ${ }^{[17]}$ and degradation of aliphatic polymers in aqueous conditions. ${ }^{[18,19]}$ Therefore, an attempt was made for the first time to synthesize an industrially important fatty 
acid ester, ${ }^{[20]}$ starch oleate using the native lipase from Cryptococcus sp. in an aqueous medium at ambient temperature. The parameters influencing the esterification process were analyzed and the product was characterized by Fourier transform infrared (FTIR) spectroscopy, Nuclear magnetic resonance (NMR) spectroscopy, X-ray diffraction (XRD), Scanning electron microscopy (SEM), and Thermogravimetric analysis (TGA). The synthesized starch oleate was also assessed for its emulsification activity.

\section{Materials and Methods}

\subsection{Materials}

The yeast strain, Cryptococcus sp. MTCC 5455 was used for lipase production and maintained on potato dextrose agar slants at $4{ }^{\circ} \mathrm{C} .{ }^{[21]}$ Potato starch, corn starch, and all the other chemicals used were of AR grade and purchased from Hi-Media Limited (Mumbai, India). The other substrates, rice starch, and arrowroot starch were purchased from local markets in Chennai, India. Standard fatty acid methyl esters and commercial lipases, Candia rugosa (L1754-5G), Pseudomonas fluorescens (534730-10G), and porcine pancreas (L3126-100G) were procured from Sigma-Aldrich Chemical Co. (St Louis, USA).

\subsection{Enzyme}

The extracellular crude lipase obtained from Cryptococcus sp. ${ }^{[17]}$ was partially purified by ammonium sulfate precipitation $(80 \%$ saturation). The precipitate was dissolved in $0.1 \mathrm{M}$ phosphate buffer ( $\mathrm{pH}$ 7.0), dialyzed and lyophilized. The enzyme (76.67 U $\mathrm{mg}^{-1}$ of protein) thus obtained was used in esterification reactions. Lipase activity was determined by the spectrophotometric method using $p$-nitrophenyl laurate ( $p$-NPL) as substrate. ${ }^{[22]}$ One unit of lipase activity was defined as the amount of enzyme that liberates $1 \mu \mathrm{mol}$ of $p$-nitrophenol per minute under the standard assay conditions.

\subsection{Lipase Catalyzed Esterification of Starch Oleate}

Different starch substrates (potato, corn, rice, and arrowroot) were dissolved in $10 \mathrm{~mL}$ of distilled water by heating in a microwave at $80^{\circ} \mathrm{C}$ for $5 \mathrm{~min}$. The esterification reaction was carried out in series of $100 \mathrm{~mL}$ screw capped Erlenmeyer flasks containing different molar ratios of oleic acid and starch (1:3$4: 1)$ at $4 \mathrm{~g}$ of total weight. The reaction was initiated by adding $1000 \mathrm{U}$ of Cryptococcus sp. lipase and the mixture was incubated at $30^{\circ} \mathrm{C}$ for $24 \mathrm{~h}$ with shaking at $140 \mathrm{rpm}$. After completion of the reaction, the starch ester synthesized was precipitated with anhydrous ethanol followed by centrifugation at $10000 \mathrm{rpm}$ for $10 \mathrm{~min}$. The precipitate was again washed with anhydrous ethanol to remove unesterified oleic acid. The starch oleate formed was dried at $60^{\circ} \mathrm{C}$ for $3 \mathrm{~h}$. The synthesis was monitored by measuring the degree of substitution (DS) and parameters influencing the synthesis were optimized by varying the concentration of lipase (500-2500 U), solvent concentration $(0-100 \%, v / v)$, reaction temperature $\left(30-50{ }^{\circ} \mathrm{C}\right)$, and reaction time (4-36h). A control without enzyme was run in parallel under the same conditions. In addition, commercial lipases were also evaluated for the synthesis of starch oleate under optimal conditions. All the experiments were carried out in triplicates and the results were expressed as the mean \pm SD of triplicates.

\subsection{Determination of DS by Methanolysis of Starch Oleate to Methyl Oleate}

The DS value of esterified starch indicates the average number of substitutions per anhydroglucose unit (AGU) in starch ${ }^{[23]}$ and it was determined by methanolysis of starch oleate. The reaction was initiated by dissolving $30 \mathrm{mg}$ of the synthesized starch oleate and a known quantity of the internal standard tricaprylin in $1 \mathrm{~mL}$ of DMSO. Two $\mathrm{mL}$ of $0.5 \mathrm{M}$ methanolic sodium methoxide was added to the mixture and incubated in a water bath at $55^{\circ} \mathrm{C}$ for $1.5 \mathrm{~h}$ with intermittent vortexing at every $20 \mathrm{~min}$. The tubes were then cooled and $2 \mathrm{~mL}$ of distilled water and $3 \mathrm{~mL}$ of hexane were added and mixed well. The top organic phase containing the methyl esters were separated and injected into an Agilent 7820A gas chromatographic system connected to a HP-5 capillary column $(30 \mathrm{~m} \times 0.320 \mathrm{~mm} \times 0.25 \mu \mathrm{m}$; J \& W Scientific, Folsom, USA) with a flame ionization detector. The column temperature was maintained at $150^{\circ} \mathrm{C}$ for $0.5 \mathrm{~min}$, increased to $210^{\circ} \mathrm{C}$ at a rate of $5^{\circ} \mathrm{C} \mathrm{min}^{-1}$ and to $300^{\circ} \mathrm{C}$ at a rate of $10^{\circ} \mathrm{C} \mathrm{min}^{-1}$. The temperatures of the injector and detector were set at 245 and $250^{\circ} \mathrm{C}$, respectively. DS was calculated as,

$\mathrm{DS}=\left(A_{1} \times I_{0} \times R_{0} \times R_{1}\right) /\left(I_{1} \times A_{0}\right)$,

where $A_{1}$ and $I_{1}$ are the test peak areas of methyl oleate and internal standard, $A_{0}$ and $I_{O}$ are the standard peak areas of methyl oleate and internal standard, $R_{O}$ is the standard mole ratio of methyl oleate to internal standard, and $R_{1}$ is the test mole ratio of internal standard to anhydroglucose units.

\subsection{FTIR Spectroscopy}

The change in chemical structure of the starch was qualitatively analyzed using FTIR-4200 spectrometer (Jasco, Japan). FTIR spectra of the thin films of dried and finely powdered starch samples ground with potassium bromide were obtained in the wavelength range of $4000-400 \mathrm{~cm}^{-1}$.

\section{6. ${ }^{7}$ NMR Spectroscopy}

${ }^{1} \mathrm{H}$ NMR spectra of the native and modified starch was obtained using Avance III Bruker spectrometer (Switzerland), operating at $400 \mathrm{MHz}$ using deuterated DMSO as solvent (2.46 ppm).

\subsection{XRD Analysis}

The XRD patterns of the starches were recorded using an X-ray diffractometer (Rigaku MiniFlex II, Japan) at $80 \mathrm{~mA}$ and $40 \mathrm{kV}$ in 
operation. The scattering angle $(2 \theta)$ was varied from $5^{\circ}$ to $80^{\circ}$ with a step width of $0.02^{\circ}$.

\subsection{SEM Studies}

The changes in surface morphology of starch and starch oleate were observed using a FEI Quanta 200 FEG electron microscopic unit at $20 \mathrm{kV}$ accelerating voltage. The samples were mounted on an aluminum stub with a double sticky tape and sputtered with a layer of gold in vacuum before analysis.

(a)

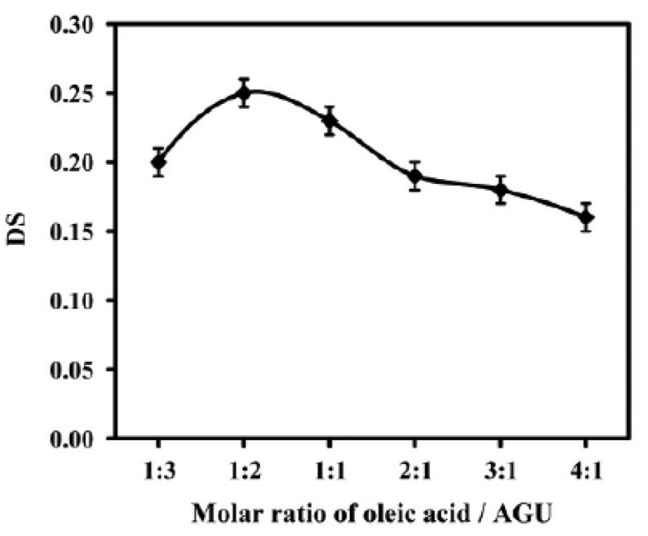

(c)

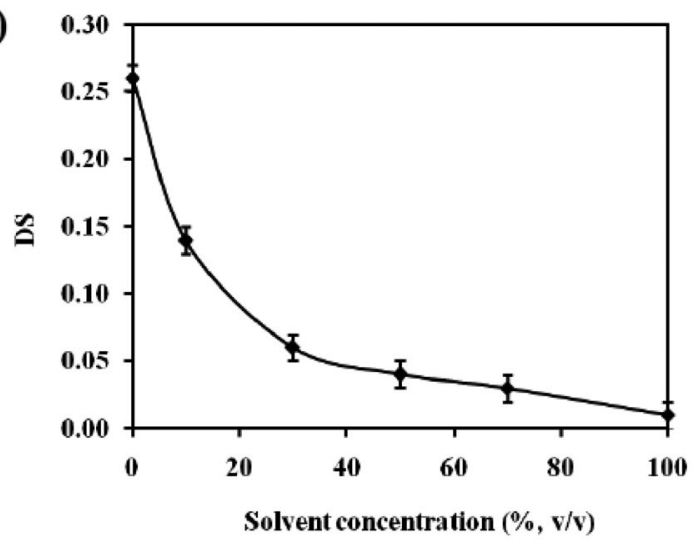

\subsection{TGA Analysis}

TGA measurements were carried out using a TGA Q50 system (TA instruments). The starch samples were heated from 30 to $800^{\circ} \mathrm{C}$ in a nitrogen atmosphere at a rate of $20^{\circ} \mathrm{C} \mathrm{min}^{-1}$.

\subsection{Emulsification Activity}

The emulsification activity of the synthesized ester was determined by gelatinizing different concentration of starch

(b)

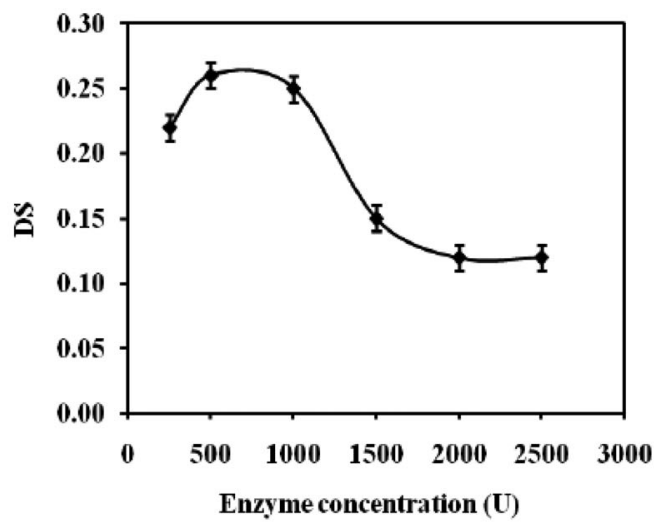

(d)

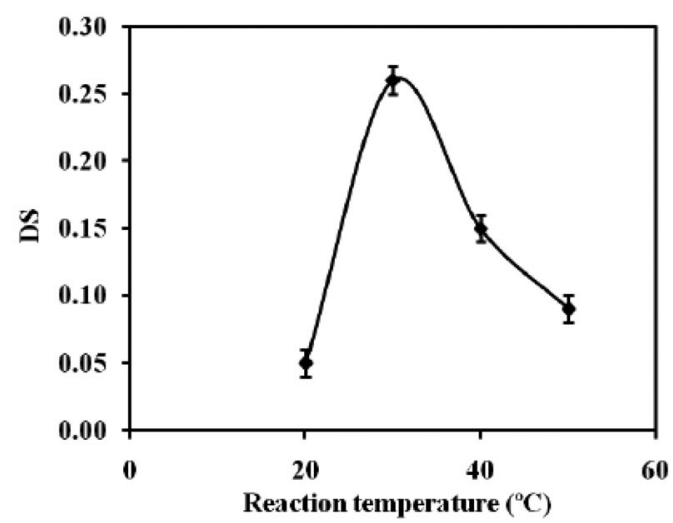

(e)

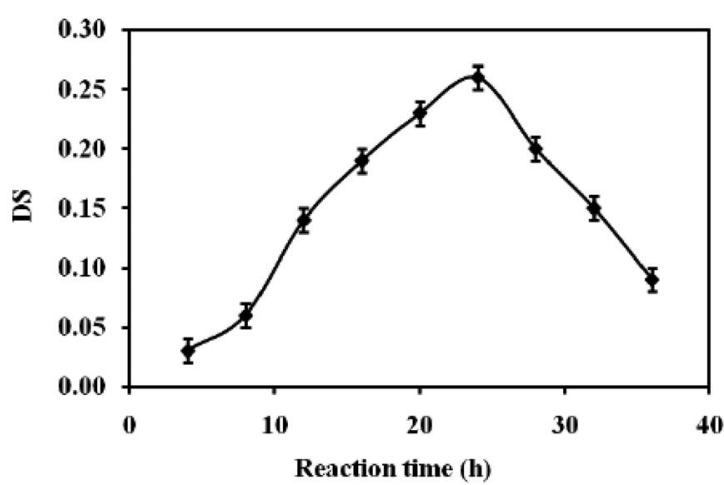

Figure 1. Effect of a) molar ratio of oleic acid/AGU, b) enzyme concentration, c) solvent concentration, d) reaction temperature, and e) reaction time on degree of substitution of starch oleate. Error bars represent standard deviations, $n=3$. 
oleate (50 and $200 \mathrm{mg}$ ) in $10 \mathrm{~mL}$ water, followed by addition of $15 \mathrm{~mL}$ heptane. The suspension was then homogenized at $10000 \mathrm{rpm}$ for $3 \mathrm{~min}$. The emulsion formed was allowed to settle and the height of the emulsion layer and the total height of liquid were measured after $1\left(t_{0}\right)$ and $24 \mathrm{~h}(t)$. The emulsion stability (ES $\%)$ corresponds to the ratio between the emulsion index (EI) at time $t$ and $t_{0}$. EI was calculated using the following equation,

$$
\begin{aligned}
\text { Emulsion index }(\mathrm{EI})= & (\text { Height of emulsion layer } \times 100) \\
& /(\text { Total height of liquid })
\end{aligned}
$$

\section{Results and Discussion}

The specific activity of crude lipase from Cryptococcus sp. ( $31.50 \mathrm{U} \mathrm{mg}^{-1}$ of protein) was 2.43 times increased by ammonium sulphate precipitation. The partially purified lipase also showed maximum activity at $\mathrm{pH} 7.0$ and at $37^{\circ} \mathrm{C}$ and was stable between $\mathrm{pH}$ 5.0 and 9.0 and at temperatures up to $50^{\circ} \mathrm{C}$ as that of the purified enzyme reported by Kamini et al. ${ }^{[21]}$ The concentrated enzyme was used for the synthesis of starch oleate and the conditions affecting the esterification reaction was determined.

\subsection{Influence of Reaction Parameters on DS of Starch Oleate}

Among different starch substrates, the DS (0.25) was higher with potato starch using molar ratio of oleic acid/AGU, 1:2. Hence, the effect of increasing concentrations of oleic acid/AGU was investigated with potato starch (Figure 1a). It was observed that the molar ratio of $4: 1$ caused a $36 \%$ decrease in DS due to steric hindrance of the existing oleate groups on starch oleate molecules, preventing further esterification ${ }^{[24]}$ and depletion of lipase in the reaction. ${ }^{[11]}$ Previous reports also indicated the usage of similar molar ratio for the synthesis of starch decanoate ${ }^{[12]}$ and starch laurate. ${ }^{[13]}$ Addition of lipase concentration above $500 \mathrm{U}$ to the reaction mixture did not show any marked impact on the DS due to the attainment of equilibrium between the substrate and catalyst (Figure 1b).

The solvent dimethyl sulfoxide is mostly used in the synthesis of starch esters owing to its ablility to solubilise starch and make it more reactive toward esterification. ${ }^{[7,25]}$ Hence, the effect of addition of different concentration of DMSO to the esterification reaction was analyzed. Addition of DMSO caused $84.62 \%$ reduction in DS at $50 \%(\mathrm{v} / \mathrm{v})$ concentration compared to the medium without solvent (Figure 1c). This could be due to the breakage of intramolecular associations in the enzyme, disrupting secondary structure, and unfolding of protein at higher concentrations of DMSO as reported by Alissandratos and Halling. ${ }^{[26]}$ Therefore, an aqueous medium without the addition of organic solvent was selected for the synthesis of starch oleate by Cryptococcus sp. lipase, while Xing et al. ${ }^{[27]}$ attained optimal esterification efficiency for synthesis of starch maleate only with $12.1 \%$ water content.

Reaction temperature plays a crucial role in DS of starch esters because it affects the activity of lipase and the structure of substrate molecule. High temperature loosens the structure of starch and enhances the movement of the molecules,
Table 1. Comparison of degree of substitution of starch oleate by Cryptococcus sp. lipase and other commercial lipases.

\begin{tabular}{lc}
\hline Source of lipase & Degree of substitution \\
\hline Candida rugosa & $0.005 \pm 0.0002$ \\
Pseudomonas fluorescens & $0.200 \pm 0.001$ \\
Porcine pancreas & $0.019 \pm 0.001$ \\
Cryptococcus sp. & $0.260 \pm 0.002$ \\
\hline
\end{tabular}

Values are means of three replicates \pm standard deviations.

contributing to the contact among the reaction groups, resulting in increased DS. ${ }^{[28]}$ In the case of Cryptococcus sp. lipase, increase in reaction temperature from 20 to $30^{\circ} \mathrm{C}$ led to rise in DS of products from 0.05 to 0.26 (Figure 1d), whereas further increase of temperature adversely affected the DS. This could be due to the inactivation of lipase at higher temperature, which was concurrent with the study of Xu et al., ${ }^{[3]}$ wherein the synthesis of octenyl succinic anhydride starch esters by Novozym 435 was optimum at $40^{\circ} \mathrm{C}$ and the DS values decreased with further increase in reaction temperature. The results indicated that synthesis of starch oleate by Cryptococcus sp. lipase could be carried out at ambient temperatures $\left(30^{\circ} \mathrm{C}\right)$, while in contrast, higher temperatures of $40,44,60$, and $160^{\circ} \mathrm{C}$ were reported by Adak and Banerjee, ${ }^{[29]}$ Horchani et al., ${ }^{[30]}$ Zarski et al., ${ }^{[31]}$ and Kshirsagar and Singhal, ${ }^{[20]}$ respectively.

A reaction time (Figure 1e) of $24 \mathrm{~h}$ was required for maximum collisions to take place between the substrates and the DS was reduced by $23.08 \%$ at $12 \mathrm{~h}$. However, prolonged incubation times decreased the DS due to hydrolysis of starch oleate and partial deactivation of the enzyme. ${ }^{[28]}$ An optimal DS of 0.26 was obtained at $24 \mathrm{~h}$ and $30^{\circ} \mathrm{C}$ with oleic acid and starch in the molar ratio of 1:2 using $500 \mathrm{U}$ of Cryptococcus sp. lipase in 100\% aqueous medium.

Commercial lipases, C. rugosa, Pseudomonas fluorescens, and porcine pancreas were compared for the synthesis of starch oleate under the optimal conditions standardized for Cryptococcus sp. lipase and the DS of starch oleate obtained were $0.05,0.200$, and 0.019 , respectively (Table 1 ). These lipases

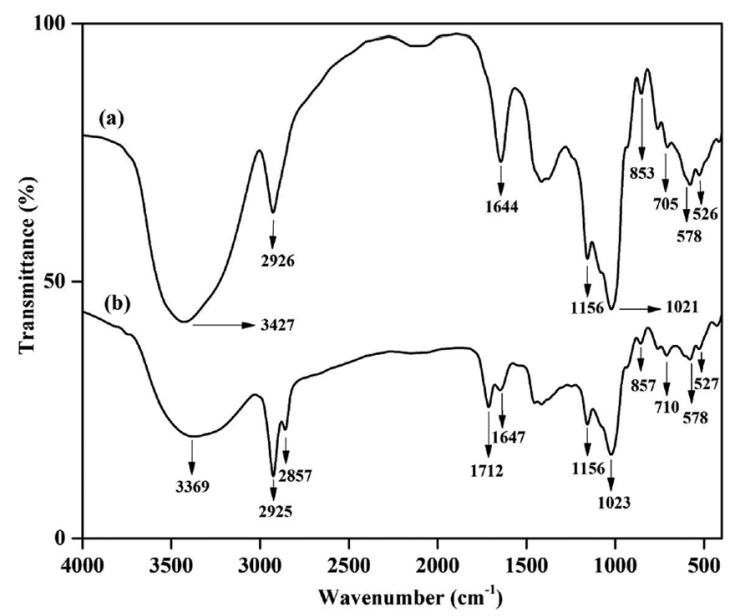

Figure 2. FTIR spectra of a) starch and b) starch oleate. 
were not found to be suitable for synthesis of starch oleate in aqueous conditions and at ambient temperature. However, lipases from C. rugosa, Pseudomonas sp., and porcine pancreas could efficiently catalyze the synthesis of starch palmitate in mixed ionic liquids (DS -0.144$),{ }^{[11]}$ hydrophobically modified starch in DMSO/dimethylformamide/dimethylacetamide
(DS -0.0052$)^{[10]}$ and starch laurate in a low moisture media $(\mathrm{DS}-0.0151),{ }^{[13]}$ respectively, at higher temperatures $\left(60^{\circ} \mathrm{C}\right)$. Moreover, the enzyme cost of commercial $C$. rugosa and $P$. fluorescens lipase is $84.59 \%$ and $83.87 \%$ higher than the partially purified lipase of Cryptococcus sp. Hence, Cryptococcus sp. lipase is unique and provides an economically feasible option for large

(a)

Figure 3. NMR spectra of a) starch and b) starch oleate. 
(a)

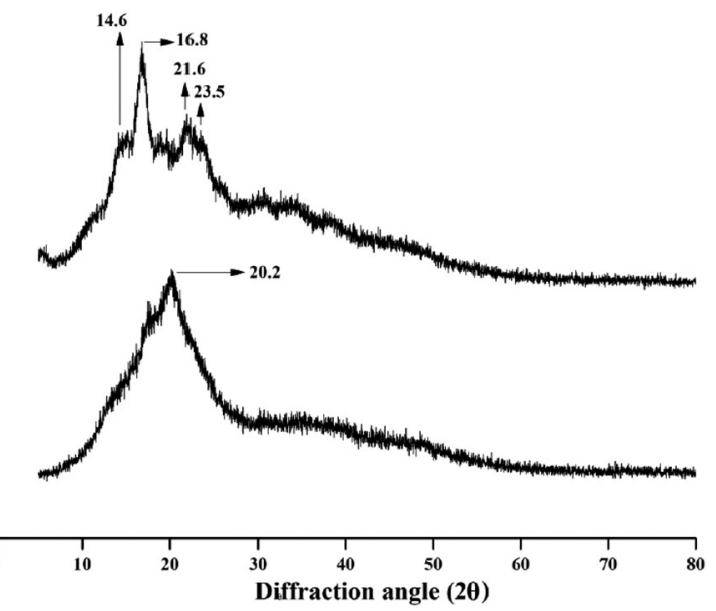

Figure 4. XRD spectra of a) starch and b) starch oleate.

scale synthesis of starch esters in an aqueous medium at ambient temperatures.

\subsection{FTIR Analysis}

In the infrared spectrum of starch and starch oleate (Figure 2), the peaks around 1156 and $1021 \mathrm{~cm}^{-1}$ were due to the stretching vibration of $\mathrm{C}-\mathrm{O}$ bonds. Several additional characteristic absorption bands also appeared around 853, 705, 578, and $526 \mathrm{~cm}^{-1}$ due to the stretching vibrations of the entire anhydroglucose ring as reported by Han et al. ${ }^{[28]}$ The peak in the region of $1659-1639 \mathrm{~cm}^{-1}$ was due to the tightly bound water present in starch and decreased in intensity after esterification. ${ }^{[5]}$ In the spectrum of starch oleate (Figure 2b), the decrease in intensity of the broad signal at $3600-3100 \mathrm{~cm}^{-1}$ corresponding to the $\mathrm{O}-\mathrm{H}$ groups of starch showed that the alcoholic groups of the polysaccharide reacted during the esterification reaction. The increase in intensity around $2926 \mathrm{~cm}^{-1}$ was due to the $\mathrm{C}-\mathrm{H}$ stretching band of oleic acid on esterification. ${ }^{[30]}$ The presence of new bands at 1712 and $2857 \mathrm{~cm}^{-1}$ was attributed to the stretching of carbonyl group $(\mathrm{C}=\mathrm{O})$ and methylene $\left(\mathrm{CH}_{2}\right)$, which confirmed that starch reacted with oleic acid to form ester carbonyl groups.

\subsection{NMR Analysis}

${ }^{1} \mathrm{H}$ NMR spectra of starch and starch oleate is shown in Figure 3. The signals corresponding to the anhydroglucose units appeared between $3.28-5.54 \mathrm{ppm}$. The signal at $5.05 \mathrm{ppm}$ was assigned to $\mathrm{H}-1,3.28 \mathrm{ppm}$ to $\mathrm{H}-2,3.67 \mathrm{ppm}$ to $\mathrm{H}-3$, and $3.52 \mathrm{ppm}$ to $\mathrm{H}-5$ protons present in starch. The hydroxyl groups of the unsubstituted starch unit resonate at 4.68, 5.42, and $5.54 \mathrm{ppm}$. In starch oleate spectrum (Figure 3b), the peak corresponding to the terminal methyl group of the acyl chain appeared at $0.78 \mathrm{ppm}$. The peaks of methylene present besides the carbonyl group and the one directly before the first methylene group were observed at 2.12 and $1.41 \mathrm{ppm}$, respectively. The resonance at $1.17 \mathrm{ppm}$ related to all the other methylene groups of the acyl chain. ${ }^{[11]}$ The results indicated the
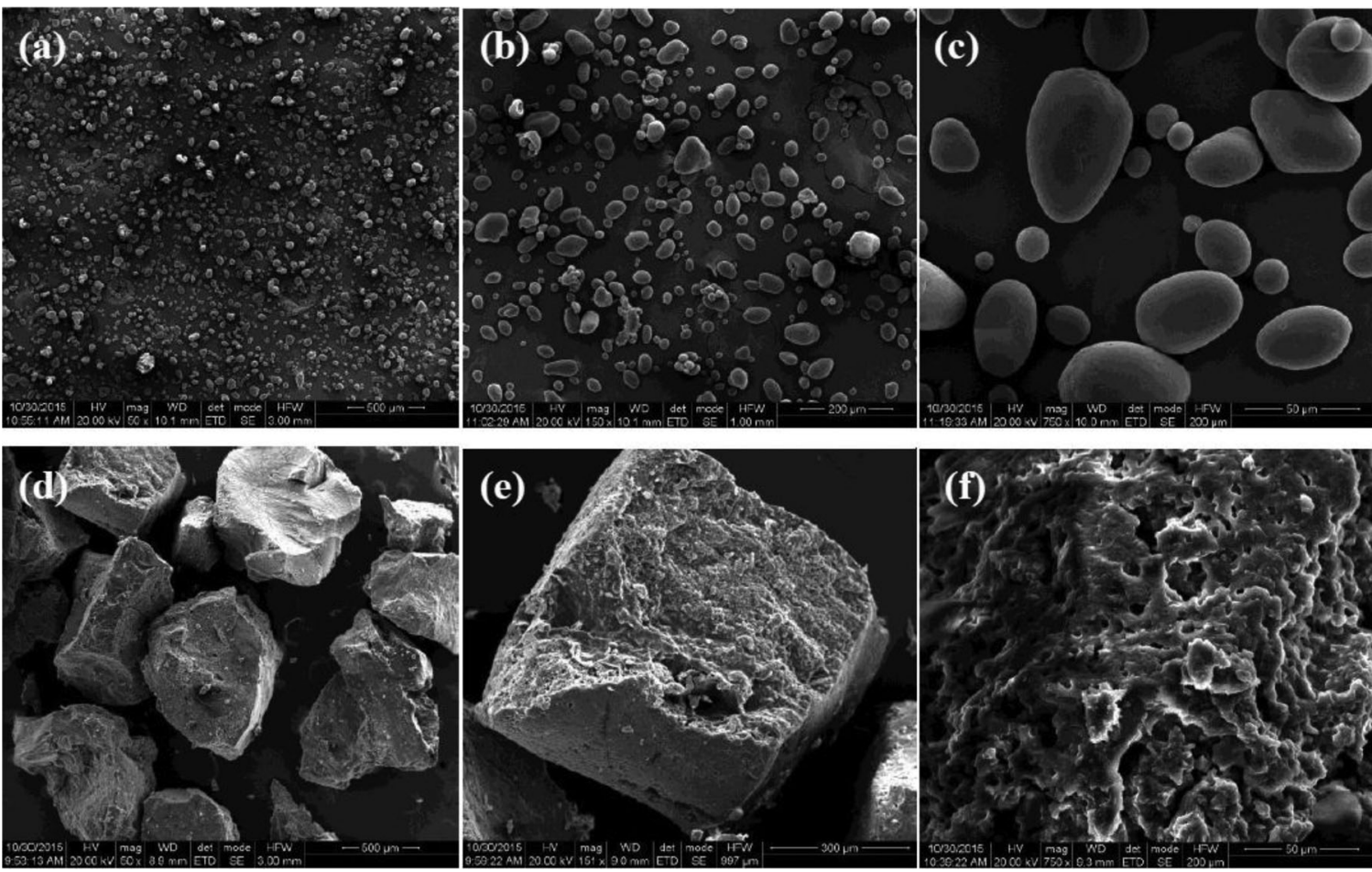

Figure 5. SEM image of a) starch $\times 50$, b) starch $\times 150$, c) starch $\times 750$, d) starch oleate $\times 50$, e) starch oleate $\times 150$, and f) starch oleate $\times 750$. 
incorporation of oleic acid groups to starch during esterification by Cryptococcus sp. lipase.

\subsection{XRD Analysis}

XRD was performed to analyze the changes in the crystallinity pattern of starch and starch oleate (Figure 4). The starch granules exhibited a typical A-type X-ray pattern with reflections $(2 \theta)$ at about 14.6, 16.8, 21.6, 23.5 (Figure 4a). After esterification, the oleate groups partially replaced the hydroxyl groups on starch and destabilized inter- and intra-molecular hydrogen bonds. The original double helix structure of starch was disrupted and a peak appeared at $20.2^{\circ}$ in starch oleate (Figure $4 \mathrm{~b}$ ). This suggested that crystallinity of starch was damaged on esterification and the molecular structure changed from a double helix pattern to a V- type single helix pattern. Similar changes in structure of acetylated and propionylated corn starch were reported by Han et al. ${ }^{[28]}$ and Tupa et al. ${ }^{[32]}$

\subsection{SEM Analysis}

The morphology of starch and starch oleate samples were investigated by SEM (Figure 5). Round or oval shaped starch granules of varying sizes with well-defined edges were observed (Figure 5a-c). In comparison to the unmodified starch, starch oleate granules were completely disrupted with loss in its individuality and smoothness (Figure 5d-f). They exhibited rough surfaces with apparent pores and cavities (Figure $5 \mathrm{f}$ ). The granules aggregated due to the damage of the ordered crystalline structure of starch on esterification. ${ }^{[24]}$ This fusion of granules was related to an increase of hydrogen bond due to the presence of carbonyl group in the newly formed esters. ${ }^{[3]}$

\subsection{Thermal Analysis}

The TGA (Figure 6a) and differential thermogravimetric (DTG) (Figure 6b) thermograms of the starch samples were acquired to study the changes in thermal stability and to determine the percent weight loss of the materials on heating. A weight loss of $10 \%$ was observed at $100^{\circ} \mathrm{C}$ for starch due to the removal of physically adsorbed and hydrogen bond linked water molecules. However, starch oleate showed only $4 \%$ loss at $100^{\circ} \mathrm{C}$, which confirmed the reduction in starch hydrophilicity owing to the replacement of some hydroxyl groups by less hydrophilic oleate groups. ${ }^{[6]}$ The mass loss was higher in starch and starch oleate between 223 and
$412{ }^{\circ} \mathrm{C}(68 \%)$ and $153-436{ }^{\circ} \mathrm{C}(71 \%)$, respectively. The decrease of initial degradation temperature in starch oleate was due to the disintegration of intramolecular hydrogen bond interactions in the starch matrices during esterification. ${ }^{[3]}$ The maximum decomposition temperature calculated from DTG data for starch and starch oleate were 333 and $329^{\circ} \mathrm{C}$, respectively. The decrease in thermal stability of starch oleate was due to the transformed structure of starch from semi crystalline to an amorphous form, as confirmed by the XRD and SEM analysis. ${ }^{[34]}$ This observation was in accordance with the reports of Desalegn et al., ${ }^{[9]} \mathrm{Gao}$

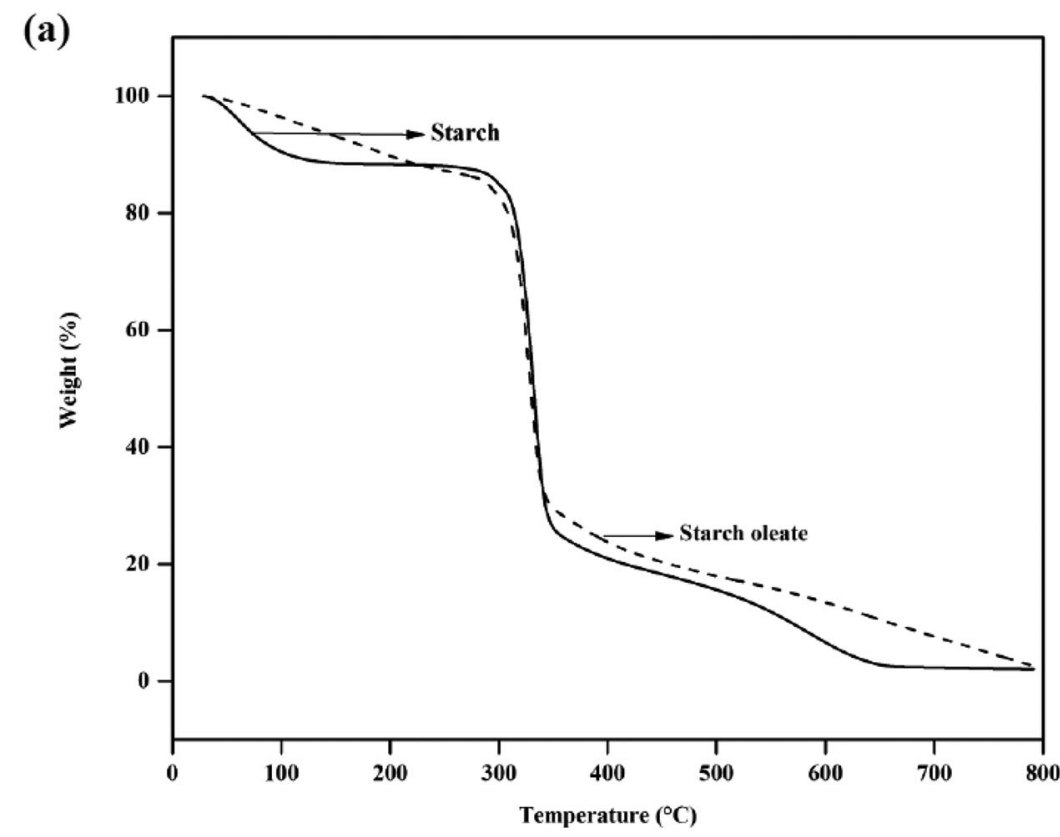

(b)

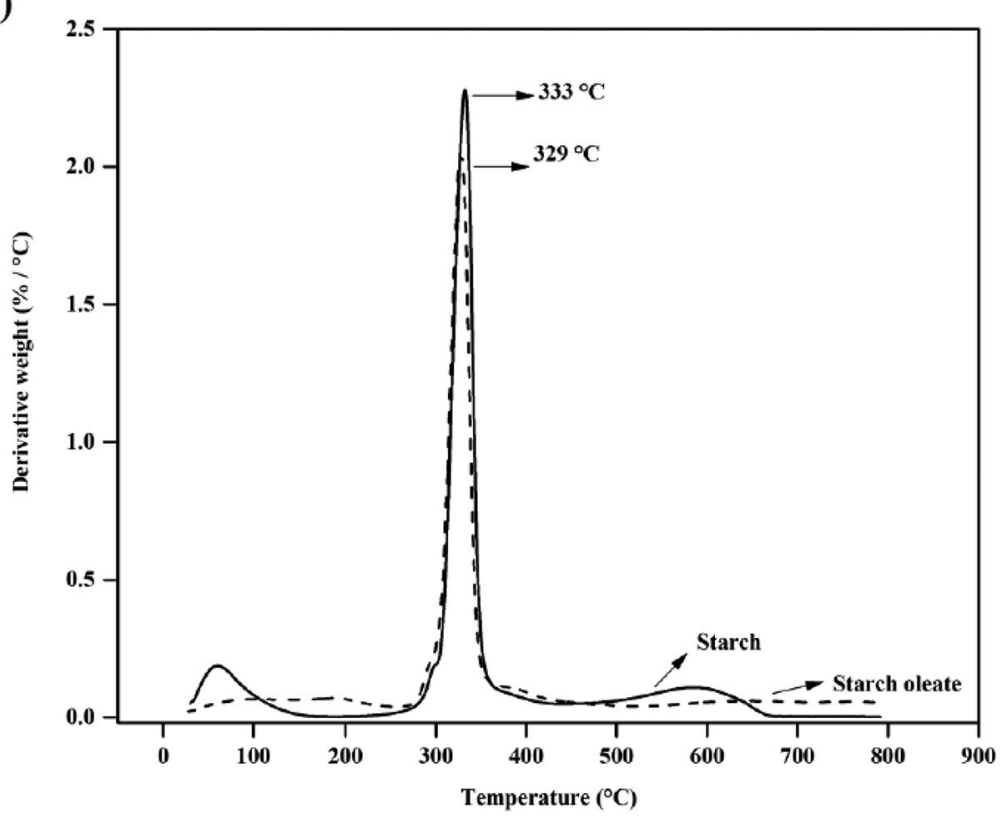

Figure 6. a) TGA and b) DTG of starch and starch oleate. 
Table 2. Emulsion stability of starch and starch oleate.

\begin{tabular}{lcccc}
\hline Sample & Concentration [mg] & El after 1h [\%] & El after 24h [\%] & ES [\%] \\
\hline Starch & 50 & $5 \pm 1$ & $2 \pm 1$ & $40 \pm 1$ \\
& 200 & $4 \pm 1$ & $2 \pm 1$ & $50 \pm 1$ \\
DS $=0.260$ & 50 & $59 \pm 2$ & $46 \pm 2$ & $78 \pm 2$ \\
& 200 & $58 \pm 2$ & $47 \pm 2$ & $81 \pm 2$ \\
DS $=0.142$ & 50 & $65 \pm 3$ & $55 \pm 3$ & $85 \pm 3$ \\
& 200 & $65 \pm 3$ & $54 \pm 3$ & $83 \pm 3$ \\
DS $=0.018$ & 50 & $24 \pm 2$ & $14 \pm 2$ & $58 \pm 2$ \\
& 200 & $29 \pm 3$ & $15 \pm 3$ & $52 \pm 3$ \\
\hline
\end{tabular}

Values are means of three replicates \pm standard deviations.

et al., ${ }^{[13]}$ Lin et al., ${ }^{[34]}$ Lu et al., ${ }^{[11,24]}$ wherein the thermal stability of starch decreased after esterification.

\subsection{Emulsification Activity}

The introduction of hydrophobic fatty acid groups in starch imparts stability to emulsions ${ }^{[35]}$ and makes it viable for industrial applications. ${ }^{[36,37]}$ Accordingly, starch oleate was synthesized and evaluated for its EI and ES at two different concentrations (Table 2). It could be observed that increasing the concentration of starch oleate did not have any marked impact on the EI and $50 \mathrm{mg}$ was found to be optimum. However, a $74.55 \%$ increase in EI was obtained at $24 \mathrm{~h}$, when the DS of starch oleate increased from 0.018 to 0.142 indicating the net hydrophobicity of the sample, thereby improving the interaction between the substrate and the oil/solvent phase. But, further increase in DS (0.260) caused a $16.36 \%$ decrease in EI at $24 \mathrm{~h}$. This might be attributed to the folding of the oleic acid chain toward the centre of the starch granule, which decreased the interaction between the solvent phase and hydrophobic chain. ${ }^{[38]}$ A maximum emulsion stability of $85 \%$ was attained using $50 \mathrm{mg}$ starch oleate with a DS of 0.142 . The results indicated that synthesis of starch oleate by Cryptococcus sp. lipase in an aqueous medium enables its use as an emulsifier in the food sector.

\section{Conclusion}

In this study, a green process was developed for the synthesis of starch oleate using Cryptococcus sp. lipase. The proposed method suggests the possibility of producing starch esters in an aqueous medium at ambient temperatures. Esterification of potato starch with oleic acid in the molar ratio of 1:2 yielded a maximum DS of 0.26 at $24 \mathrm{~h}$ and $30^{\circ} \mathrm{C}$. The presence of chemically bound fatty acid chains in the esterification products were verified by FTIR and ${ }^{1} \mathrm{H}$ NMR spectroscopy. Changes in crystalline pattern and decrease in thermal stability of starch was also observed on esterification. The results also unearthed a new facet of the Cryptococcus sp. lipase in the food sector, due to the fact that the synthesized ester could render $85 \%$ stability to emulsions. Therefore, the enzymatically produced starch oleate could be used as an ingredient in food products where viscosity and hydrophobic interactions are desired.

\section{Acknowledgements}

The authors thank Director, CSIR-CLRI, Chennai, India, for his kind permission to publish this work. The financial support extended by the Council of Scientific and Industrial Research, New Delhi, India to M.A. Aarthy is gratefully acknowledged.

\section{Conflict of Interest}

The authors declare no conflict of interest.

\section{Keywords}

aqueous media, Cryptococcus sp. lipase, emulsifiers, potato starch, starch oleate

Received: November 23, 2017

Revised: May 11, 2018

Published online:

[1] Y. Zhang, T. Gan, H. Hu, Z. Huang, A. Huang, Y. Zhu, Z. Feng, M. Yang, Ind. Eng. Chem. Res. 2014, 53, 2114.

[2] J. Hong, R. Che, X. Zeng, Z. Han, Food Chem. 2016, 192, 15.

[3] J. Xu, C. Zhou, R. Wang, L. Yang, S. Du, F. Wang, H. Ruan, G. He, Carbohydr. Polym. 2012, 87, 2137.

[4] N. Prasertpornsakun, M. Raita, N. Laosiripojana, V. Champreda, Biosci. Biotechnol. Biochem. 2015, 79, 1750.

[5] A. Rajan, J. D. Sudha, T. E. Abraham, Ind. Crops Prod. 2008, 27, 50.

[6] M. Tupa, L. Maldonado, A. Vazquez, M. L. Foresti, Carbohydr. Polym. 2013, 98, 349.

[7] A. Rajan, T. E. Abraham, Bioprocess Biosyst. Eng. 2006, 29, 65.

[8] A. Biswas, R. L. Shogren, G. Selling, J. Salch, J. L. Willett, C. M. Buchanan, Carbohydr. Polym. 2008, 74, 137.

[9] T. Desalegn, I. J. V. Garcia, J. Titman, P. Licence, I. Diaz, Y. Chebude, Starch/Staerke 2014, 66, 385.

[10] L. Qiao, Q. Gu, H. N. Cheng, Carbohydr. Polym. 2006, 66, 135.

[11] X. Lu, Z. Luo, S. Yu, X. Fu, J. Agric. Food Chem. 2012, 60, 9273.

[12] A. Alissandratos, N. Baudendistel, S. L. Flitsch, B. Hauer, P. J. Halling, BMC Biotechnol. 2010, 10, 1.

[13] Y. Gao, L. Wang, X. Yue, G. Xiong, W. Wu, Y. Qiao, L. Liao, Starch/ Staerke 2014, 66, 450.

[14] C. Lecointe, E. Dubreucq, P. Galzy, Biotechnol. Lett. 1996, 18, 869.

[15] N. R. Kamini, H. lefuji, Process Biochem. 2001, 37, 405.

[16] K. Thirunavukarasu, N. G. Edwinoliver, S. D. Anbarasan, M. K. Gowthaman, H. lefuji, N. R. Kamini, Process Biochem. 2008, 43, 701.

[17] M. Aarthy, P. Saravanan, N. Ayyadurai, M. K. Gowthaman, N. R. Kamini, J. Mol. Catal., B Enzym. 2016, 125, 25.

[18] K. Thirunavukarasu, S. Purushothaman, M. K. Gowthaman, T. Nakajima-Kambe, C. Rose, N. R. Kamini, J. Food Sci. Technol. $2015,52,5772$.

[19] K. Thirunavukarasu, S. Purushothaman, J. Sridevi, M. Aarthy, M. K. Gowthaman, T. Nakajima-Kambe, N. R. Kamini, Int. Biodeterior. Biodegradation. 2016, 110, 99.

[20] A. C. Kshirsagar, R. S. Singhal, Carbohydr. Polym. 2007, 69, 455.

[21] N. R. Kamini, T. Fujii, T. Kurosu, H. lefuji, Process Biochem. 2000, 36, 317. 
[22] K. Isobe, T. Akiba, S. Yamaguchi, Agric. Biol. Chem. 1988, 52, 41.

[23] V. D. Miladinov, M. A. Hanna, Ind. Eng. Chem. Res. 1999, 38, 3892.

[24] X. Lu, Z. Luo, X. Fu, Z. Xiao, J. Agric. Food Chem. 2013, 61, 9882.

[25] P. Ptaszek, M. Lukasiewicz, A. Ptaszek, M. Grzesik, J. Skrzypek, M. Kulawska, Starch/Staerke 2011, 63, 181.

[26] A. Alissandratos, P. J. Halling, Bioresour. Technol. 2012, 115, 41.

[27] G. Xing, S. B. Zhang, J. Yang, Starch/Staerke 2006, 58, 464.

[28] F. Han, C. Gao, M. Liu, F. Huang, B. Zhang, Int. J. Biol. Macromol. 2013, 59, 372.

[29] S. Adak, R. Banerjee, Carbohydr. Polym. 2016, 150, 359.

[30] H. Horchani, M. Chaabouni, Y. Gargouri, A. Sayari, Carbohydr. Polym. 2010, 79, 466.

[31] A. Zarski, S. Ptak, P. Siemion, J. Kapusniak, Carbohydr. Polym. 2016, 137, 657.
[32] V. M. Tupa, S. Arroyo, M. L. Herrera, M. L. Foresti, Starch/Staerke 2018, 70, 1.

[33] E. Hermawan, L. Rosyanti, L. Megasari, A. K. Sugih, H. Muljana, Int. J. Chem. Eng. Appl. 2015, 6, 152.

[34] R. Lin, H. Li, H. Long, J. Su, W. Huang, Biomed Res. Int. 2014, 2014, 1.

[35] L. A. Bello-Perez, C. A. Bello-Flores, M. C. Nunez-Santiago, C. P. Coronel-Aguilera, J. Alvarez-Ramirez, Carbohydr. Polym. 2015, 132, 17.

[36] Y. V. Garcia-Tejeda, E. J. Leal-Castaneda, V. Espinosa-Solis, V. Barrera-Figueroa, Carbohydr. Polym. 2018, 194, 177.

[37] L. F. Hong, L. H. Cheng, C. Y. Gan, C. Y. Lee, K. K. Peh, LWT- Food Sci. Technol. 2018, 91, 526.

[38] A. Viswanathan, J. Environ. Polym. Degrad. 1999, 7, 191. 